\title{
Research Progress in the Study of Startle Reflex to Disease States
}

\author{
Junfeng Zhang ${ }^{1,2}$, Meng Wang', Baoyu Wei ${ }^{3}$, Jiangwei Shi', Tao Yu' \\ 'First Teaching Hospital of Tianjin University of Traditional Chinese Medicine, Tianjin, 300380, People's Republic of China; ${ }^{2}$ National Clinical Research \\ Center for Chinese Medicine Acupuncture and Moxibustion, Tianjin, 300380, People's Republic of China; ${ }^{3}$ State Key Laboratory of Component-based \\ Chinese Medicine, Institute of Traditional Chinese Medicine, Tianjin University of Traditional Chinese Medicine, Tianjin, 301617, People's Republic of \\ China
}

Correspondence: Tao Yu, Email doctoryutao@163.com

\begin{abstract}
The startle reflex is considered a primitive physiological reflex, a defense response that occurs in the organism when the body feels sudden danger and uneasiness, characterized by habituation and sensitization effects, and studies on the startle reflex often deal with pre-pulse inhibition (PPI) and sensorimotor gating. Under physiological conditions, the startle reflex is stable at a certain level, and when the organism is in a pathological state, such as stroke, spinal cord injury, schizophrenia, and other diseases, the reflex undergoes a series of changes, making it closely related to the progress of disease. This paper summarizes the startle reflex in physiological and pathological states by reviewing the databases of PubMed, Web of Science, Cochrane Library, EMBASE, China Biology Medicine, China National Knowledge Infrastructure, VIP Database for Chinese Technical Periodical, Wanfang Data, and identifies and analyzes the startle reflex and excessive startle reaction disorder.
\end{abstract}

Keywords: startle reflex, disease, hyperekplexia, review

\section{Introduction}

The startle reflex in humans is a conserved systemic motion response that is ubiquitous in mammals. It is the reflex contraction of the skeletal and facial muscles to sudden intense stimuli, and includes eye blinking, limb flexion, trunk shrugs, and autonomic symptoms (such as increased heart rate, sweating). ${ }^{1}$ It originates from the brainstem, descends along the reticular tract to the spinal cord, ${ }^{2}$ and finally stimulates the muscles through motor neurons, the strength of which are measured by electromyography (EMG). ${ }^{3}$ The startle reflex is considered a defensive (relative to directional) response, which is habitual. ${ }^{5}$ As the number and frequency of startles increase, the body becomes habitual, and the degree of startle reflex decreases. This kind of reaction exists to a certain extent to protect the human body. The startle reflex is an effective tool for studying the basic characteristics of the central nervous system. It has the characteristics of crossspecies consistency, simple neural circuits, and sensitivity to experimental operations. ${ }^{5}$ Throughout our review, we found that the degree of attention to the startle reflex has increased in the past five years, but review articles were mostly limited to the habituation, sensitization, pre-pulse inhibition (PPI), and sensorimotor gating of the startle reflex. Thereafter, we focused on summarizing the startle reflex in clinical disease states, and distinguishing the startle reflex from hyperekplexia.

In this article, the truncation and Boolean logic operators were used to search the databases of PubMed, Web of Science, Cochrane Library, EMBASE, China Biology Medicine, China National Knowledge Infrastructure, VIP Database for Chinese Technical Periodical, Wanfang Data according to the combination of subject words and free words. Herein, we summarized the startle reflex in physiological and pathological states and analyzed both the reflex and excessive startle reaction disorder. The search keywords were as follows: "startle reflex," "startle response," "mechanism," "pathology," "physiology," "pre-pulse suppression," "sensorimotor gating," "disease," "rare diseases," "hyperekplexia," "stroke," "spinal cord injuries," "schizophrenia," and "autistic disorder." 


\section{Classifications}

Mammalian sensory organs are diverse; thus the startle reflex can be classified as auditory, visual, tactile, or olfactory. Among them, the most widely studied in clinical research is the auditory startle reflex (ASR), ${ }^{6}$ which may be related to the difference in the processing time of stimulation among the known sensory modalities. For example, the total processing time for sound stimuli is shorter than that for visual stimuli, possibly because of the faster conduction time in the ear and the shorter distance from the ear to the auditory cortex. The tactile startle reflex often causes a local reaction as compared to the auditory startle reflex, contributing to a certain incubation period, resulting in an atypical response; therefore, there are few related studies. ${ }^{7}$ Unless specified, the startle reflex described in this article is the auditory startle reflex. The ASR is most prominent in the facial, neck, and shoulder muscles, and rarely in the lower body. Surface EMG records obtained from the orbicularis (OOC), sternocleidomastoid (SCM), biceps brachii (BB), and tibialis anterior (TA) muscles were used as research indicators. ${ }^{8}$

\section{Measurement Methods}

The startle reflex is important when studying the input and output of brainstem information, and there are many methods for measuring it. The most common and original method for measuring the startle reflex is the EMG system. With professional training and equipment (wired sensors), EMG detects the electrical signals of the target activity, obtaining objective and relevant data on the startle reflex. Other novel measurement methods include the new optical non-invasive method, 9 mobile acoustic startle reflex monitoring system (MARS), ${ }^{10}$ and video freeze monitoring system. ${ }^{5}$ The Balogiannis team ${ }^{9}$ believe that the new optical non-invasive method is simple and reliable, making it superior to traditional EMG systems in terms of effectiveness, accuracy, and reliability. MARS ${ }^{10}$ is the first mobile phone-based monitoring system. Through the use of smartphone applications for visual recording, ASR can be detected and monitored in healthy people. The diagnosis and monitoring of ASR in stroke, traumatic brain injury, and mood disorders has opened up new prospects and horizons, and the same went for that in remote or isolated conditions. The video freeze system ${ }^{5}$ (Georgia Medical Association Co., Ltd., Vermont, USA) uses a standard digital camera to shoot videos and automatically evaluates the rodents' shock response to sound. The system is also equipped with stimuli, such as sounds and lights, that are required for various startle modes, which is a new tool for studying startle reflex. These multiple measurement methods enable more efficient and realistic evaluation of the startle reflex.

\section{Related Mechanisms \\ Sensorimotor Gating}

Sensorimotor gating and startle reflex are both important components of the brainstem reflex, and it is necessary to understand one to study the other. Sensorimotor gating is a control mechanism for the effective transmission of sensory information to the motor system. ${ }^{11}$ It usually occurs in the early stages of central nervous system processing, so that unnecessary stimuli are filtered out or partially ignored, and the central nervous system is less trivial, thereby optimizing the exchange of brain information, and efficiently using limited resources to process important information. Disruption of sensorimotor gating is prominent in many neurological and psychiatric diseases, including Parkinson's disease (PD), schizophrenia, and Tourette's syndrome.

\section{PPI}

PPI is a standard measurement method for sensorimotor gating. It is considered to be a potential biomarker reflecting brain function in disease states. ${ }^{12}$ It is a stable physiological marker of the nervous system that accompanies human growth, and develops until its full maturation at approximately 8 years of age. It is mainly mediated by the brainstem and affected by the cortex - striatum - globus pallidus - pontine systems. ${ }^{5}$ It is usually defined as a decrease in startle reflex due to weak sensation or pre-stimulus. Whenever a weaker stimulus ("pre-pulse," such as a sound of about 85 decibels) appears before a strong stimulus, it results in a reduced startle amplitude to the strong stimulus ("pulse," such as a sound of about 118 decibels). Simultaneously, PPI adjusts sensory input by filtering out irrelevant content or distracting stimuli to prevent sensory information overflow, thereby selectively and efficiently processing relevant information. ${ }^{13}$ 


\section{Neural Circuits}

At present, the neural circuits of the startle reflex are still unclear. Fang et $\mathrm{al}^{14}$ explored the neural circuit of the startle reflex by analyzing the retina- superior colliculus (SC)-lateral posterior nucleus (LP)-primary visual cortex (V1) pathway. The retina transmits visual information to the SC, which uploads information to the LP, which activates the L1 inhibitory neurons to inhibit the V1 response, and process different types of visual information. This pathway increases the incoming threshold of sensory information by screening important information, enabling the brain to receive more accurate information in the shortest time, and issuing commands in a timely manner during emergencies. ${ }^{15}$ The pulvinar nucleus of the thalamus is an important part in this pathway, and is mutually and extensively connected with the prefrontal cortex, sensory cortex, superior colliculus, and amygdala, playing a very important role in multimodal sensory processing, emotional response, and defensive behavior. ${ }^{16,17}$ In addition, abnormal pulvinar nucleus activity is associated with excessive startling. ${ }^{18}$

It is relatively simple that the primary auditory startle reflex neural pathway in mammals. External sound activates primary auditory neurons in the cochlear root and cochlear nucleus $(\mathrm{CN})$ that transmit the auditory signals to giant neurons of the caudal pontine reticular nucleus $(\mathrm{PnC})$ in the brainstem, then the $\mathrm{PnC}$ giant neurons directly activate spinal motor neurons (MNs), which finally form the CN-PnC-MNs pathway. ${ }^{19} \mathrm{PPI}$ is activated by midbrain structures primarily including the inferior colliculus (IC), superior colliculus (SC), and peduncular tegmentum (PPTg). ${ }^{20}$ The PPI pathway is also affected by midbrain and corticolimbic structures. The basolateral amygdala (BLA) activates the nucleus accumbens (NAcc), which in turn inhibits the ventral pallidus (VP), and ultimately the VP inhibits PPTg. ${ }^{21}$ The above together form the cortico-striato-pallido-pontine (CSPP) network. ${ }^{22}$ More current studies suggest that in addition to the CSPP pathway regulating the startle reflex, there are other pathways. It has been observed that the damage of the BLA leads to an excessive enhancement of the startle reflex, so the startle reflex pathway was investigated from the central nucleus of the amygdala (CeA), and a CeA-PnC pathway independent of the CSPP was found. ${ }^{23}$ This pathway does not affect the threshold level of startle reflex, but modulates the magnitude and duration of startle reflex. ${ }^{24}$ So far, studies have identified two neural pathways related to the auditory startle reflex, the CSPP neural pathway that mediates PPI and the CeA-PnC pathway that uses the lentiform nucleus as an initiation signal. In the future, more neural pathways and mechanisms may be developed.

\section{Startle Reflex in Disease States}

The strengthening or weakening of the startle reflex can reflect the pathological conditions of the body; related in-depth studies are of great significance in diagnosing and treating clinical diseases. In certain disease states, such as central nervous system traumatic diseases (traumatic brain injury, stroke), neurodegenerative diseases (Alzheimer's disease and PD), and some mental system diseases (schizophrenia), the central nervous system is reorganized, altering the startle reflex. ${ }^{6}$ For different diseases, the startle reflex is different. For neurological diseases, the startle reflex is often strengthened, which often helps patients to participate in active activities and is conducive to later recovery. For mental disorders, declining habituation of the startle reflex allows patients to maintain a high level of arousal to external stimuli, which can interfere with the body's ability to pay attention, process, and remember information.

\section{Startle Reflex After Stroke}

A quarter of stroke patients have an increased startle reflex. ${ }^{25}$ Honeycutt et al ${ }^{26}$ believed that compared with normal subjects, stroke patients have an increased startle reflex, which manifests as increased limb activity when stimulated, suggesting that the startle reflex is relevant to the recovery of motor function in patients after stroke, but whether the

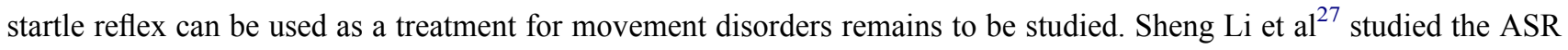
response of the damaged biceps brachii in 16 patients with post-stroke hemiplegia, and reported that the auditory evoked potential response of the injured biceps muscle showed different patterns at different stages of stroke. For patients with post-stroke spasticity, the frequency, amplitude, and duration of the auditory startle reflex increase, suggesting a connection between spasticity and startle reflex. 
The main pathway of the startle reflex is the reticulospinal tract, beginning from the medulla oblongata and pons, and running parallel to the corticospinal tract that innervates skeletal muscle movement. ${ }^{28} \mathrm{~A}$ large number of overlapping neurons in the descending pathways terminate in the gray matter layers VII and VIII of the lateral cord and anterior cord, and primarily regulate muscle tension in both limbs. ${ }^{29}$ After stroke onset, the integrity of the central nervous tissues and functions of the corticospinal tract are damaged, reducing human motor function to varying degrees. At this time, the compensatory excitement of the reticular spinal tract ${ }^{30}$ can replace the corticospinal cord to a certain extent, which also helps to recover movement after stroke. In contrast, it can also lead to spasticity, ${ }^{31}$ confirming the important role of the reticulospinal tract in spasticity. Therefore, the startle reflex can be used as a tool to monitor the excitability of the reticulospinal tract. ${ }^{32}$ The whole process of post-stroke exercise recovery can be divided broadly into three main stages, and subdivided into six stages according to Brunnstrom as follows: fistula soft stage (stage I), spasticity stage (stages IIIV), and chronic recovery stage (stages V-VI), with the startle reflex different in each stage. ${ }^{33}$ In the soft stage, the startle reflex exists as normal, suggesting an intact reticular spinal cord pathway; in the spastic period, the startle reflex is exaggerated, indicating increased excitability of the reticulospinal tract; in the recovery period, the startle reflex is reduced, indicating reduced compensatory excitement of the spinal tract, and restored motor function of the corticospinal tract. The extent of exaggeration of the reflex in ther spastic phase depends on the severity and location of the sports injury. ${ }^{34}$

The startle reflex pathway plays different roles in different stages of exercise recovery, especially for spasticity. The startle reflex pathway can be used to develop a personalized rehabilitation plan for each Brunnstrom stage to promote exercise recovery. It is also a follow-up point when studying spastic development after stroke onset.

\section{Startle Reflex After Spinal Cord Injury}

Spinal cord injury (SCI) can cause changes in the functions of the central and peripheral nervous systems, ${ }^{35}$ such as increase excitability above the lesion, ${ }^{36}$ and damage to the bottom segmental motor neuron of the corticospinal tract and injury plane. The possibility of interrupted neuron regeneration after SCI is extremely small. At this time, functional recovery usually requires reorganization of multiple levels of the central nervous system and backup nerve pathways. ${ }^{37}$ Reorganization can occur at multiple levels, including the cerebral cortex, brainstem, or spinal cord, ${ }^{38}$ as well as different neural pathways. Aside from the corticospinal tract, the human motor nervous system has five other descending conduction pathways as follows: red nucleus, vestibular, tectorial, reticular, and medial longitudinal tracts. ${ }^{37}$ After SCI, the corticospinal tract is damaged; thus, the other normal brainstem spinal cord bundles initiate a compensatory mechanism, and the enhancement of the startle reflex also indicates reorganization of the reticulospinal pathway. ${ }^{39}$ At this time, the startle reflex can be used to monitor the reorganization of neural pathways in SCI patients.

Regarding whether the degree of enhancement of the startle reflex is related to the damaged plane and degree of SCI, the probability of ASR in patients with SCI was higher than that in normal subjects. ${ }^{39}$ The incubation period of ASR in patients with high-level SCI is significantly shorter than that in patients with low-level SCI, but the difference in ASR latency was not statistically significant between complete and incomplete SCI patients. The number of cervical SCI is greater than that of thoracolumbar SCIs; hence, the reorganization and plasticity of the central nervous system are more obvious. Therefore, the degree of enhancement of the startle reflex may be related to the number of damaged neuronal reorganizations, rather than the extent of the disease. After SCI, the reticular spinal cord is reorganized, and the enhanced startle reflex can guide the treatment and prognosis; simultaneously, it can increase the patients' belief in rehabilitation, as suggested by Kumru. ${ }^{7}$ The exaggerated stimulus response, especially the auditory startle reflex, may help enhance the voluntary movement of patients with SCI and facilitate the effective development of later rehabilitation training.

\section{Startle Reflex in Schizophrenia}

Schizophrenia is a psychiatric disorder characterized by impairments in the integration of information and cognition, including thinking, distraction, and information processing deficits, and affects approximately $1 \%$ of the world's population. ${ }^{5}$ However, its etiology is not well understood. After onset, the level of sensorimotor gating in the brain gradually decreases. The existence of this mechanism is to filter the influence of unnecessary information on the human body. When sensorimotor gating is reduced, an increase in unnecessary interference will stimulate the symptoms of 
patients with schizophrenia, which is generally regarded as a measure of responsiveness to external environmental stimuli, and it represents a method of studying reactivity and habituation. One physiological mechanism which is lacking in schizophrenia is PPI, which is modulated by the cortico-striatal-pallidal-pontine pathway and involved neurotransmitters. ${ }^{5}$

There are different opinions about whether the startle reflex in patients with schizophrenia is decreased or increased. Existing studies divide the users into three drug groups according to use: typical antipsychotics, atypical antipsychotics, and control. Most studies ${ }^{40}$ reported a reduced startle reflex in the typical antipsychotic group. Psychiatric drugs (such as clozapine) can alleviate the difference between patients with schizophrenia and normal subjects, proving the effect of drugs on schizophrenia. However, some previous studies ${ }^{41}$ report conflicting results. Other studies believe that patients with schizophrenia experience different emotions, thereby exhibiting different startle reflexes. When the subjects are in a state of disgust or anxiety, the degree of startle increases, and when they are in a delusional state, their shock amplitude is significantly higher. However, in patients with mainly negative symptoms, particularly flat emotions, their ASR sensitivity may be lower, possibly because of the weakened ability of these patients to express emotional stimuli. ${ }^{42}$ Therefore, the startle reflex is generally regarded as a measure of the response to external environmental stimuli. Research on the startle reflex will help to understand schizophrenia and help standardize clinical medication regimens.

\section{Startle Reflex for Other Diseases}

Many diseases can cause changes in the startle reflex of the human body. These changes help us understand the pathogenesis and principles of the disease. The startle reflex can also be used as a detection tool to guide clinical treatment and prognosis.

The latency of the ASR in children with autism spectrum disorder (ASD) is prolonged, and the PPI level decreases, which may be related to the impaired adaptive function of children with autism in multiple areas, including social, communication, occupation, or other important areas of daily life. ${ }^{43}$ Impaired adaptability can easily lead to an increased risk of adverse behaviors in such patients.

One of the earliest clinical symptoms of Tay-Sachs disease is the enhanced startle reflex. ${ }^{44}$ The brains of patients with Tay-Sachs disease show an enhanced stem electrophysiological response, suggesting that the enhanced startle reflex is related to changes in the physiological state of the brainstem. In addition, augmented startle reflex characteristics of TaySachs disease are also observed in GM1 gangliosidosis, metachromatic leukodystrophy, and Krabbe disease. ${ }^{45}$ These diseases need to be distinguished, and brainstem research should be strengthened.

Regarding the relationship between myoclonus and brain stem reticular structure in patients with progressive myoclonic epilepsy (PME), the frequency of ASR in PME patients was significantly reduced, the average latency was longer, and the reduced startle reflex suggested that the reticular structures were less excitable, which is attributable to atrophy and degenerative changes. ${ }^{46-48}$

Yavuz et $\mathrm{al}^{49}$ found that the neural pathways of ASR in patients with coexisting essential tremor and Parkinson's disease (ET-PD) with obvious dyskinesia were significantly abnormal, while the ASR in patients with pure essential tremor (TE) or PD was similar to that in healthy subjects, indicating that ET-PD is a unique disease, and cortical and brainstem structural abnormalities show close associations.

Urbach-Wiethe disease is a rare genetic disorder that causes near-complete calcification of the basolateral amygdala (BLA). The study of a single individual ${ }^{50}$ confirmed that when the BLA function is impaired, the patient's learning ability for challenging tasks is weaker than that of the startle reflex. The slower learning system is not enough to quickly stimulate the defensive response, whereas the excessive startle reflex to fear stimuli will be detrimental to the patient's emotional stability. This suggests that the BLA is essential for coordinating the adverse effects and effective changes produced by excessive startle.

Startle Reflex Modulation Measurement can be used to objectively assess indicators in patients with Alzheimer's disease, which is considered the only objective physiological measure of emotion that is sensitive to emotional valence. ${ }^{51}$ For patients with Alzheimer's disease, especially those in the advanced stage, it is almost impossible to participate in daily living activities through accurate verbal expression and physical behavior. Using the startle reflex can summarize 
their emotional responses to various stimuli. ${ }^{52}$ This process can connect the patient with the surrounding environment, giving nursing workers or family members more useful information to facilitate high-quality patient care.

\section{Difference Between Startle Reflex and Hyperekplexia}

Hyperekplexia is easily confused with the startle reflex. The startle reflex is the symmetrical contraction response of the body when the body is stimulated, whereas hyperekplexia is a rare neurological disorder caused by abnormal glycinergic neurotransmission which usually develops in the neonatal period, with genetic predisposition. ${ }^{53}$ When faced with sudden visual, auditory, or tactile stimuli, patients with hyperekplexia experience jitters, tremors, stiffness, and other phenomena. In severe cases, it may suffocate and require resuscitation to save lives. Older children often fall down after repeated attacks, while physical examination showed increased muscle tone and positive nose percussion tests.

The disease is similar to reflex epilepsy, abnormal brain development, tetanus, convulsions, and other diseases. Hyperekplexia patients may simultaneous develop reflex epilepsy; ${ }^{54}$ therefore, abnormal electroencephalography (EEG) during the ictal period cannot be used to diagnose epilepsy alone. If the startle response symptoms persist despite antiepileptic drug intake, hyperekplexia may be considered, and genetic testing can be performed to confirm the diagnosis. For diseases that are easily misdiagnosed, EEG and other brain imaging examinations are conducive to early differential diagnosis, and gene sequencing can confirm the diagnosis. After the startle reaction, the abdominal pressure can be increased, resulting to umbilical hernia, inguinal hernia, and hip dislocation. ${ }^{55}$ Clonazepam has a good effect on this disease. Tao Dongying et $\mathrm{al}^{56}$ found that clinical symptoms in children significantly improved after taking low-dose clonazepam for 2 days, the muscle stiffness disappeared, and no falls occurred again. The Vigevano strategy can alleviate attacks and reduce mortality. ${ }^{57,58}$ However, the specific diagnosis and treatment of this disease still depends on individual conditions, and there is no clear and systematic treatment plan.

The triggering conditions of the startle reflex are similar to those of hyperekplexia, both of which are caused by the sudden stimulation of the human body by the external environment, such as sound, light, and touch. The phenomenon is similar, and the body contraction response exists; however, hyperekplexia is a disease other than contraction. Additionally, there are other phenomena, such as shaking, tremors, and stiffness, which may even affect life. The startle reflex can occur at all ages, and will change accordingly under different diseases, whereas hyperekplexia is an independent neurological disease that frequently occurs in newborns have a complete disease system of "conceptgenesis-diagnosis-differential diagnosis-treatment-prognosis". In clinical practice, attention should be paid to differentiation between the two.

\section{Conclusion}

As a defensive response of the human body, the startle reflex is an important tool to check whether the excitability of the brainstem network structure is normal and whether the nerve conduction pathway is intact. It is of great value to study disease pathogenesis, diagnosis, treatment, and prognosis. The highlights of this review include: (1) PPI is a measure of sensorimotor gating, which can be used as a starting point for research on central nervous system diseases. So far, studies have identified two neural pathways related to the auditory startle reflex, the CSPP neural pathway and the CeA-PnC pathway. In future research, more neural pathways and mechanisms may be developed; (2) the startle reflex is enhanced for patients with post-stroke spasticity, suggesting that the compensatory effect of the reticulospinal tract on the corticospinal tract may be beneficial to the recovery of patients after stroke; (3) there is no difference in the startle reflex between patients with complete and incomplete SCI, and cervical SCIs are more reorganized than thoracoc SCIs, while the startle reflex in patients with lumbar SCIs is enhanced, suggesting that the reorganization of the nervous system after spinal cord injury may be related to the level of injury, and not the extent; (4) the startle reflex is enhanced in schizophrenia patients, so the startle reflex can be used as a criterion for evaluating the efficacy of schizophrenia drugs; (5) the study of the startle reflex in rare diseases helps to understand its pathogenesis, clinical treatment, and prognosis; (6) the startle reflex is a physiological or pathological phenomenon in mammals, whereas hyperekplexia is a genetic pathology commonly seen in newborns. 
In conclusion, the startle reflex has important guiding value for studying various stages of various diseases. There is still plenty of room for research on the startle reflex. Afferent pathways in the brainstem are different, and so are other types of startle reflexes. Randomized controlled studies are needed to fully understand the startle reflex.

\section{Abbreviations}

EMG, electromyography; PPI, prepulse inhibition; CBM, China Biology Medicine; CNKI, China National Knowledge Infrastructure; VIP, VIP Database for Chinese Technical Periodical; ASR, acoustic startle reflex; OOC, orbicularis; SCM, sternocleidomastoid; BB, biceps brachii; TA, tibialis anterior; MARS, mobile acoustic startle reflex monitoring system; PD, Parkinson's Disease; SC, superior colliculus; LP, lateral posterior nucleus; V1, Primary Visual Cortex; CN, cochlear nucleus; PnC, caudal pontine reticular nucleus; MNs, motor neurons; IC, inferior colliculus; PPTg, pedunculopontine tegmental nucleus; BLA, Basolateral amygdaloid nucleus, anterior part; NAcc, nucleus accumbens; VP, ventral pallidus; CSPP, cortico-striato-pallido-pontine; CeA, Central amygdaloid nucleus; SCI, spinal cord injuries; ASD, Autism Spectrum Disorder; PME, progressive myoclonic epilepsy; ET-PD, Coexisting Essential Tremor Parkinson's Disease; ET, essential tremor; BLA, basolateral amygdala; EEG, electroencephalography.

\section{Author Contributions}

All authors made a significant contribution to the work reported, whether that is in the conception, study design, execution, acquisition of data, analysis and interpretation, or in all these areas; took part in drafting, revising or critically reviewing the article; gave final approval of the version to be published; have agreed on the journal to which the article has been submitted; and agree to be accountable for all aspects of the work.

\section{Funding}

This work was supported by the financial support from the National Natural Science Foundation of China (No. 81503672).

\section{Disclosure}

The authors report no conflicts of interest in this work.

\section{References}

1. Chen YT, Li S, Zhou P, Li S. A startling acoustic stimulation (SAS)-TMS approach to assess the reticulospinal system in healthy and stroke subjects. J Neurol Sci. 2019;399:82-88. doi:10.1016/j.jns.2019.02.018

2. McPherson JG, Chen A, Ellis MD, Yao J, Heckman CJ, Dewald JPA. Progressive recruitment of contralesional cortico-reticulospinal pathways drives motor impairment post stroke. J Physiol. 2018;596(7):1211-1225. doi:10.1113/JP274968

3. Brownstone RM, Chopek JW. Reticulospinal systems for tuning motor commands. Front Neural Circuits. 2018;12:30. doi:10.3389/ fncir.2018.00030

4. Gómez-Nieto R, Hormigo S, López DE. Prepulse inhibition of the auditory startle reflex assessment as a hallmark of brainstem sensorimotor gating mechanisms. Brain Sci. 2020;10(9):639.

5. Pantoni MM, Herrera GM, Van Alstyne KR, Anagnostaras SG. Quantifying the acoustic startle response in mice using standard digital video. Front Behav Neurosci. 2020;14:83. doi:10.3389/fnbeh.2020.00083

6. Kumru H, Vidal J, Kofler M, Benito J, Garcia A, Valls-Solé J. Exaggerated auditory startle responses in patients with spinal cord injury. $J$ Neurol. 2008;255(5):703-709. doi:10.1007/s00415-008-0780-3

7. Ertuglu LA, Aydin A, Kumru H, et al. Jendrassik maneuver effect on spinal and brainstem reflexes. Exp Brain Res. 2019;237(12):3265-3271. doi:10.1007/s00221-019-05668-y

8. McPherson JG, McPherson LM, Thompson CK, Ellis MD, Heckman CJ, Dewald JPA. Altered neuromodulatory drive may contribute to exaggerated tonic vibration reflexes in chronic hemiparetic stroke. Front Hum Neurosci. 2018;9(12):131. doi:10.3389/fnhum.2018.00131

9. Balogiannis G, Stachtea X, Yova D, Papageorgiou C. A novel optical non-intrusive method for measuring acoustic startle reflex in humans. Physiol Meas. 2020;41(6):065003. doi:10.1088/1361-6579/ab8e60

10. Gowen CL, Khwaounjoo P, Cakmak YO. EMG-free monitorization of the acoustic startle reflex with a mobile phone: implications of sound parameters with posture related responses. Sensors (Basel). 2020;20(21):5996. doi:10.3390/s20215996

11. MacLaren DA, Markovic T, Clark SD. Assessment of sensorimotor gating following selective lesions of cholinergic pedunculopontine neurons. Eur J Neurosci. 2014;40(10):3526-3537. doi:10.1111/ejn.12716

12. Miller EA, Kastner DB, Grzybowski MN, Dwinell MR, Geurts AM, Frank LM. Robust and replicable measurement for prepulse inhibition of the acoustic startle response. Mol Psychiatry. 2020;6:10. 
13. Azzopardi E, Louttit AG, DeOliveira C, Laviolette SR, Schmid S. The role of cholinergic midbrain neurons in startle and prepulse inhibition. $J$ Neurosci. 2018;38(41):8798-8808. doi:10.1523/JNEUROSCI.0984-18.2018

14. Fang Q, Chou XL, Peng B, Zhong W, Zhang LI, Tao HW. A differential circuit via retino-colliculo-pulvinar pathway enhances feature selectivity in visual cortex through surround suppression. Neuron. 2020;105(2):355-369.e6. doi:10.1016/j.neuron.2019.10.027

15. Ibrahim LA, Mesik L, Ji XY, et al. Cross-modality sharpening of visual cortical processing through layer-1-mediated inhibition and disinhibition. Neuron. 2016;89(5):1031-1045. doi:10.1016/j.neuron.2016.01.027

16. Zhou N, Masterson SP, Damron JK, Guido W, Bickford ME. The mouse pulvinar nucleus links the lateral extrastriate cortex, striatum, and amygdala. $J$ Neurosci. 2018;38(2):347-362. doi:10.1523/JNEUROSCI.1279-17.2017

17. Chou XL, Fang Q, Yan L, et al. Contextual and cross-modality modulation of auditory cortical processing through pulvinar mediated suppression. Elife. 2020;6(9):e54157. doi:10.7554/eLife.54157

18. Renaud D, Brodsky M. GM2-Gangliosidosis, AB variant: clinical, ophthalmological, MRI, and molecular findings. JIMD Rep. 2016;25:83-86.

19. Li L, Du Y, Li N, Wu X, Wu Y. Top-down modulation of prepulse inhibition of the startle reflex in humans and rats. Neurosci Biobehav Rev. 2009;33(8):1157-1167. doi:10.1016/j.neubiorev.2009.02.001

20. Meng Q, Ding Y, Chen L, Li L. The medial agranular cortex mediates attentional enhancement of prepulse inhibition of the startle reflex. Behav Brain Res. 2020;6(383):112511. doi:10.1016/j.bbr.2020.112511

21. Fulcher N, Azzopardi E, De oliveira C, et al. Deciphering midbrain mechanisms underlying prepulse inhibition of startle. Prog Neurobiol. 2020;185:101734. doi:10.1016/j.pneurobio.2019.101734

22. Naysmith LF, Kumari V, Williams SCR. Neural mapping of prepulse-induced startle reflex modulation as indices of sensory information processing in healthy and clinical populations: a systematic review. Hum Brain Mapp. 2021;42(16):5495-5518. doi:10.1002/hbm.25631

23. Cano JC, Huang W, Fénelon K. The amygdala modulates prepulse inhibition of the auditory startle reflex through excitatory inputs to the caudal pontine reticular nucleus. BMC Biol. 2021;19(1):116. doi:10.1186/s12915-021-01050-z

24. Hitchcock J, Davis M. Lesions of the amygdala, but not of the cerebellum or red nucleus, block conditioned fear as measured with the potentiated startle paradigm. Behav Neurosci. 1986;100(1):11-22. doi:10.1037/0735-7044.100.1.11

25. Li S, Bhadane M, Gao F, Zhou P. The reticulospinal pathway does not increase its contribution to the strength of contralesional muscles in stroke survivors as compared to ipsilesional side or healthy controls. Front Neurol. 2017;8:627. doi:10.3389/fneur.2017.00627

26. Honeycutt CF, Perreault EJ, Draganski B. Planning of ballistic movement following stroke: insights from the startle reflex. PLoS One. 2012;7(8): e43097. doi:10.1371/journal.pone.0043097

27. Li S, Chang SH, Francisco GE, Verduzco-Gutierrez M. Acoustic startle reflex in patients with chronic stroke at different stages of motor recovery: a pilot study. Top Stroke Rehabil. 2014;21(4):358-370. doi:10.1310/tsr2104-358

28. Li S, Chen YT, Francisco GE, Zhou P, Rymer WZ. A unifying pathophysiological account for post-stroke spasticity and disordered motor control. Front Neurol. 2019;10:468. doi:10.3389/fneur.2019.00468

29. Chen YT, Li S, DiTommaso C, Zhou P, Li S. Possible contributions of ipsilateral pathways from the contralesional motor cortex to the voluntary contraction of the spastic elbow flexors in stroke survivors: a TMS Study. Am J Phys Med Rehabil. 2019;98(7):558-565. doi:10.1097/ PHM.0000000000001147

30. Ellis MD, Carmona C, Drogos J, Dewald JPA. Progressive abduction loading therapy with horizontal-plane viscous resistance targeting weakness and flexion synergy to treat upper limb function in chronic hemiparetic stroke: a Randomized Clinical Trial. Front Neurol. 2018;9:71. doi:10.3389/ fneur.2018.00071

31. Chen YT, Li S, Magat E, Zhou P, Li S. Motor overflow and spasticity in chronic stroke share a common pathophysiological process: analysis of within-limb and between-limb EMG-EMG coherence. Front Neurol. 2018;9:795. doi:10.3389/fneur.2018.00795

32. Choudhury S, Shobhana A, Singh R, et al. The relationship between enhanced reticulospinal outflow and upper limb function in chronic stroke patients. Neurorehabil Neural Repair. 2019;33(5):375-383. doi:10.1177/1545968319836233

33. Li S. Spasticity, motor recovery, and neural plasticity after stroke. Front Neurol. 2017;8:120. doi:10.3389/fneur.2017.00120

34. Li S, Francisco GE. New insights into the pathophysiology of post-stroke spasticity. Front Hum Neurosci. 2015;9:192. doi:10.3389/ fnhum.2015.00192

35. Jianping J, Shengdi C. Neurology [M]. 4th ed. Beijing: China Medical Publishing House; 2018:173-182.

36. Abanoz Y, Abanoz Y, Gündüz A, et al. Pattern of startle reflex to somatosensory stimuli changes after spinal cord injury. J Spinal Cord Med. 2018;41(1):36-41. doi:10.1080/10790268.2016.1211580

37. Fink KL, Cafferty WB. Reorganization of intact descending motor circuits to replace lost connections after injury. Neurotherapeutics. 2016;13 (2):370-381. doi:10.1007/s13311-016-0422-x

38. Nardone R, Höller Y, Brigo F, et al. Functional brain reorganization after spinal cord injury: systematic review of animal and human studies. Brain Res. 2013;1504:58-73. doi:10.1016/j.brainres.2012.12.034

39. Gündüz A, Uzun N, Örnek Nİ, Ünalan H, Karamehmetoğlu ŞS, Kızıltan ME. Trigemino-cervical reflex in spinal cord injury. Neurosci Lett. 2014;580:169-172. doi:10.1016/j.neulet.2014.08.006

40. Wang Z, Tan Y, Yang F, et al. The study of acoustic startle reflex in male patients with chronic schizophrenia. Asian J Psychiatr. $2012 ; 5(1): 54-57$. doi:10.1016/j.ajp.2011.12.010

41. Swerdlow NR, Light GA, Cadenhead KS, Sprock J, Hsieh MH, Braff DL. Startle gating deficits in a large cohort of patients with schizophrenia: relationship to medications, symptoms, neurocognition, and level of function. Arch Gen Psychiatry. 2006;63(12):1325-1335. doi:10.1001/ archpsyc.63.12.1325

42. Haß K, Bak N, Szycik GR, Glenthøj BY, Oranje B. Deficient prepulse inhibition of the startle reflex in schizophrenia using a cross-modal paradigm. Biol Psychol. 2017;128:112-116. doi:10.1016/j.biopsycho.2017.07.016

43. Ebishima K, Takahashi H, Stickley A, Nakahachi T, Sumiyoshi T, Kamio Y. Relationship of the acoustic startle response and its modulation to adaptive and maladaptive behaviors in typically developing children and those with autism spectrum disorders: a Pilot Study. Front Hum Neurosci. 2019;13:5. doi:10.3389/fnhum.2019.00005

44. Zhang J, Chen H, Kornreich R, Yu C. Prenatal diagnosis of tay-sachs disease. Methods Mol Biol. 2019;1885:233-250.

45. Cachon-Gonzalez MB, Zaccariotto E, Cox TM. Genetics and Therapies for GM2 Gangliosidosis. Curr Gene Ther. 2018;18(2):68-89. doi:10.2174/ 1566523218666180404162622 
46. Lingan J. A case of $\mathrm{KCNC1}$ gene mutation-related progressive myoclonic epilepsy [A]. Chinese Anti-Epilepsy Association EEG and Neuroelectrophysiology Branch. The 7th CAAE EEG and Neuroelectrophysiology Conference Proceedings [C]; $2020 ; 2$.

47. Holmes GL. Drug treatment of progressive myoclonic epilepsy. Paediatr Drugs. 2020;22(2):149-164. doi:10.1007/s40272-019-00378-y

48. Kızıltan ME, Gündüz A, Coşkun T, et al. Startle response in progressive myoclonic epilepsy. Clin EEG Neurosci. 2017;48(2):123-129. doi:10.1177/1550059416646292

49. Yavuz D, Gündüz A, Ertan S, et al. Specific brainstem and cortico-spinal reflex abnormalities in coexisting essential tremor and Parkinson's disease (ET-PD). Neurophysiol Clin. 2015;45(2):143-149. doi:10.1016/j.neucli.2015.01.001

50. Chew LJ, Fusar-Poli P, Schmitz T. Oligodendroglial alterations and the role of microglia in white matter injury: relevance to schizophrenia. Dev Neurosci. 2013;35(2-3):102-129. doi:10.1159/000346157

51. Lyons G, Arthur-Kelly M, Eidels A, Mavratzakis A. Deep assessment: a novel framework for improving the care of people with very advanced alzheimer's disease. Biomed Res Int. 2015;2015:749451. doi:10.1155/2015/749451

52. Jafari Z, Kolb BE, Mohajerani MH. Prepulse inhibition of the acoustic startle reflex and P50 gating in aging and Alzheimer's disease. Ageing Res Rev. 2020;59:101028. doi:10.1016/j.arr.2020.101028

53. Horváth E, Farkas K, Herczegfalvi A, Nagy N, Széll M. Identification of a novel missense GLRA1 gene mutation in hyperekplexia: a case report. $J$ Med Case Rep. 2014;8:233. doi:10.1186/1752-1947-8-233

54. Sohtaoğlu M, Kızıltan E, Gündüz A, Bozluolçay M. Startle responses after different stimulus modalities differ in stroke. Neurophysiol Clin. 2016;46(3):193-199. doi:10.1016/j.neucli.2015.12.010

55. Mine J, Taketani T, Yoshida K, et al. Clinical and genetic investigation of 17 Japanese patients with hyperekplexia. Dev Med Child Neurol. 2015;57 (4):372-377. doi:10.1111/dmcn.12617

56. Dongying T, Guilian S, Zhiliang Y, Fang Y, Qianzhong Z, Hong J. Clinical and molecular genetic analysis of a case of Hyperekplexia[J]. Chin J Pract Pediatr. 2018;33(12):945-946.

57. Luat AF, Kamat D, Sivaswamy L. Paroxysmal nonepileptic events in infancy, childhood, and adolescence. Pediatr Ann. 2015;44(2):e18-23. doi:10.3928/00904481-20150203-07

58. Orivoli S, Facini C, Pisani F. Paroxysmal nonepileptic motor phenomena in newborn. Brain Dev. 2015;37(9):833-839. doi:10.1016/j. braindev.2015.01.002

\section{Publish your work in this journal}

Neuropsychiatric Disease and Treatment is an international, peer-reviewed journal of clinical therapeutics and pharmacology focusing on concise rapid reporting of clinical or pre-clinical studies on a range of neuropsychiatric and neurological disorders. This journal is indexed on PubMed Central, the 'PsycINFO' database and CAS, and is the official journal of The International Neuropsychiatric Association (INA). The manuscript management system is completely online and includes a very quick and fair peer-review system, which is all easy to use. Visit http://www.dovepress.com/testimonials.php to read real quotes from published authors.

Submit your manuscript here: https:/www.dovepress.com/neuropsychiatric-disease-and-treatment-journal 